\title{
Comparative effects of sevoflurane and propofol based general anaesthesia for elective surgery on memory
}

Lito Flouda ${ }^{1}$, Ageliki Pandazi ${ }^{1}$, Charalampos Papageorgiou², Despoina Perrea ${ }^{3}$, Eleni Krepi', Georgia Kostopanagiotou ${ }^{1}$ $12^{\text {nd }}$ Department of Anaesthesiology, Attikon University Hospital of Athens, Athens,
Greece
$21^{\text {st }}$ Department of Psychiatry, Aiginiteion University Hospital of Athens, Athens, Greece
3Laboratory of Experimental Surgery, National and Kapodistrian University of Athens,
Athens, Greece

Submitted: 30 November 2011

Accepted: 11 March 2012

Arch Med Sci 2013; 9, 1: 105-111

DOI: 10.5114 /aoms.2013.33351

Copyright (c) 2013 Termedia \& Banach

\author{
Corresponding author: \\ Ageliki Pandazi MD, PhD \\ $2^{\text {nd }}$ Department \\ of Anaesthesiology \\ Attikon University Hospital \\ of Athens \\ Rimini 1, GR-12462 \\ Chaidari, Greece \\ Phone: +302105832371 \\ Fax: +30.210 .5326413$ \\ E-mail: angpant@med.uoa.gr
}

\begin{abstract}
Introduction: Unconscious processing of words during general anaesthesia has been suggested. We used the process dissociation procedure (PDP) to test memory performance during sevoflurane and propofol anaesthesia in relation to hypnotic depth.

Material and methods: One hundred participants anaesthetised for elective surgery (50 with propofol and 50 with sevoflurane) and 50 non-anaesthetized listened to a list of words. The bispectral index (BIS) of the anaesthetised patients was recorded. Within $36 \mathrm{~h}$ after word presentation, memory was assessed using a word stem completion task, based on Buchner's model applied on the PDP. Results: There was evidence of memory for words presented during light (BIS $61-80)(p=0.001)$ and adequate (BIS 41-60) ( $p=0.008)$ but not deep anaesthesia (BIS 21-40) ( $p=0.09)$. The PDP showed a significant implicit but not explicit memory contribution (mean total explicit memory scores: $0.04 \pm 0.07$ in all BIS categories; mean implicit memory scores: $0.01 \pm 0.04,0.1 \pm 0.08$, and 0.05 \pm 0.09 at BIS $=21-40,41-60$, and 61-80, respectively). There was a statistically significant difference between the mean implicit memory score $(\mathrm{I})$ of the propofol and sevoflurane group in the BIS category 41-60 in general $(p=0.016)$, and after incision $\left(\mathrm{I}_{\mathrm{A} . \mathrm{I}}\right)(p=0.005)$ in particular, with propofol depressing I more than sevoflurane in both cases. Memory performance of nonanaesthetized participants was better, with a higher contribution of explicit and a comparable contribution of implicit memory.

Conclusions: During general anaesthesia, implicit memory persists even in adequate hypnotic states. Sevoflurane affects the implicit memory of adequately anaesthetised subjects less than propofol.
\end{abstract}

Key words: implicit/explicit memory, process dissociation procedure, bispectral index.

\section{Introduction}

Explicit memory refers to the conscious recollection of previous experiences, whereas implicit memory refers to changes in performance or behaviour produced by previous experiences. Anaesthetic drugs act prominently on explicit memory [1, 2]. Older studies suggest that explicit memory (awareness) during general anaesthesia can occur, but it is rare (0.1-0.2\%) and is often due to an inadequate level of anaesthesia [3-5]. 
The effects on implicit memory have not been studied so extensively. Whether implicit retrieval of information presented during general anaesthesia can also occur remains controversial in the literature [6-8], which may be explained by the lack of standardization of various study variables [9].

Most studies have used the process dissociation procedure (PDP) to evaluate the preservation of memory during general anaesthesia and to distinguish between explicit and implicit retrieval processes [10-13]. The PDP in combination with Buchner's extended model is considered a reliable model for memory assessment [14]. The results have been conflicting. Some studies using propofol suggest the preservation of implicit memory during general anaesthesia [15, 16] and others not [14, 17-19]. There are a few studies about the effects of sevoflurane on implicit memory. Renna's study suggests the preservation of implicit memory only for end tidal sevoflurane concentrations lower than 1.2\% [20]. Kerssen's et al.'s [21] and Stonnel's et al.'s [22] studies suggest the preservation of implicit memory during adequate general anaesthesia, whereas Kristoffer's et al.'s study [23] suggests the suppression of perceptual learning during anaesthesia with sevoflurane. We did not, however, find any studies comparing sevoflurane's and propofol's effect on memory.

Our aim was to evaluate explicit and implicit memory performance during general anaesthesia with propofol and sevoflurane for elective surgery using the PDP. The question was whether memory preservation was related to the anaesthetic agent, the depth of anaesthesia, and the surgical stimulus.

\section{Material and methods}

\section{Participants}

After approval by the ethics committee of Attikon University Hospital and written informed consent from each participant, 100 participants, aged 18-60 years, ASA I-III, undergoing elective surgery under general anaesthesia, were included in the study. The patients were randomly allocated to group A (receiving sevoflurane) or B (receiving propofol for maintenance of anaesthesia). Another 50 non-anaesthetised volunteers were also included in our study conducted from November 2006 to November 2008.

Participants who were not native Greek speakers, had a known hearing deficit, a neurological/psychiatric pathology or those under treatment affecting the cognitive function, suffering from memory dysfunction, head trauma, chronic alcoholism/drug abuse, or programmed for cardiac, obstetric or brain surgery were excluded from the study. The nonanaesthetised volunteers had similar characteristics and the same inclusion and exclusion criteria as the anaesthetised participants but were noninstitutionalized.
On the day before surgery, the participants were informed that they would listen to some words through headphones during the operation and after several hours, they would undergo an unspecified test. The auditory material for the memory test (word stem completion task) consisted of 60 threesyllable Greek words chosen according to a pilot word stem completion study [24].

\section{Procedure}

This was a double blind, randomised, clinical trial. All anaesthetised participants were unpremedicated and randomly assigned to one of the two anaesthetic regimens using sevoflurane or propofol as the main anaesthetic. They all received propofol and fentanyl for induction of anaesthesia. Participants of group A received sevoflurane (Et Sevoflurane $1.2 \%$ ) and those of group $B$ propofol infusion (50-100 $\mu \mathrm{g} / \mathrm{kg} / \mathrm{min}$ ) combined with fentanyl, a neuromuscular blocking agent and an air-oxygen mixture for maintenance of anaesthesia.

Standard monitoring including electrocardiogram, pulse oximeter, and non-invasive blood pressure was applied to all participants. A BIS A- $2000^{\circledR}$ monitor (Aspect Medical Systems Inc., Newton, MA) with single-use silver-silver chloride electrodes (Zipprep; Aspect Medical Systems) was attached to all participants' foreheads through a single channel. The bispectral index (BIS) was recorded every $15 \mathrm{~s}$ on a portable computer. Mean arterial blood pressure $(\mathrm{BP})$, heart rate $(\mathrm{HR})$, oesophageal temperature $\left(\mathrm{T}_{\text {central }}\right)$, peripheral oxygen saturation $\left(\mathrm{SatO}_{2}\right)$, and end-tidal concentrations of $\mathrm{CO}_{2}\left(\mathrm{EtCO}_{2}\right)$ were also recorded every $1.5 \mathrm{~min}$. Each participant heard two practice words in order to adjust the volume. The program for word presentation started as soon as possible after induction of anaesthesia.

The administration of anaesthesia and necessary deviations from the standard protocol were the responsibility of the anaesthesiologist (who was different from the researcher). All personnel were blinded to BIS indications. Anaesthesia was titrated using classic clinical signs (blood pressure and heart rate variation, sudation, movements, eyes opening, grimacing, cough, and pupil diameter). Participants who accidentally received benzodiazepines were excluded from the study.

In the presentation phase, two lists of 20 words each, one for the inclusion and one for the exclusion condition of the PDP were played to the participants via headphones placed on their ears, connected to a laptop (Sony VAIO VGN-FZ21S, 2005 Sony Corporation, made in China). The program chose the word lists according to a counterbalancing scheme (Table I) and presented the words in random order, different for each participant. Each word was repeated 25 times consecutively, with a 2second delay between repetitions. We allotted 
a period of $2 \mathrm{~s}$ to each word. A new word presentation started $5 \mathrm{~s}$ after the end of the previous presentation. The entire set of auditory stimuli lasted $70 \mathrm{~min}$.

The mean values of BIS and the other parameters (BP, $\mathrm{HR}, \mathrm{T}_{\text {central }}$, SatO ${ }_{2}$, EtCO ${ }_{2}$ ) were automatically computed and averaged for each presented word. If the BIS value for any given word was at any time above 60 we replaced the average BIS with the maximal recorded BIS for the particular word.

The same task was administered to the nonanaesthetised volunteers in a quiet room. They were instructed to listen carefully to a series of repeated words that would be included in a later unspecified test.

For the evaluation phase we used the PDP originally introduced by Jacoby [25] in combination with Buchner's et al.'s extended model [26]. The evaluation was performed within $36 \mathrm{~h}$ after the presentation phase (retention interval) both on non-anaesthetised and anaesthetised participants, whenever they felt comfortable, and the retention interval was recorded for each one. Each previously anaesthetised participant was first given a short interview roughly testing preservation of explicit memory during general anaesthesia [27]:

1. What is the last thing you remember before you fell asleep?

2. What is the first thing you remember after you woke up?

3. Do you remember any words or any intraoperative events?

4. Do you remember having any dreams and if so what were they about?

In the evaluation phase, each participant was presented with three lists of word stems according to the group he/she was originally assigned. Thirty word stems were presented in each condition, 20 corresponding to previously presented target words and 10 to not previously heard distractor words, in order to assess the completion rate due to chance or guessing (base rate). These stems were audible only to the participants.

In the inclusion condition, measuring general memory performance, participants were asked to use each stem as a clue to recall the previously heard word or otherwise complete the stem with the first 3-syllable word that came to mind. According to Buchner's model the subject's performance in this condition is described by equation (1): Inclusion $=e+(1-e) \cdot[i+(1-i) \cdot g]$. Parameter $e$ represents explicit memory, $i$ implicit memory, and $g$ the base rate in the inclusion condition.

In the exclusion condition, measuring implicit effect, the participants were instructed to use the stem as a clue to recall a word previously heard, then avoid this word and find another one. The participant's performance in this condition is described by Buchner's
Table I. Counterbalancing scheme for words used in the process dissociation procedure

\begin{tabular}{|lccc|}
\hline Group & $\begin{array}{c}\text { List 1 } \\
\text { (words 1-20) }\end{array}$ & $\begin{array}{c}\text { List 2 } \\
\text { (words 21-40) }\end{array}$ & $\begin{array}{c}\text { List 3 } \\
\text { (words 41-60) }\end{array}$ \\
\hline 1 & Inclusion target & Exclusion target & Distractor \\
\hline 2 & Distractor & Inclusion target & Exclusion target \\
\hline 3 & Exclusion target & Distractor & Inclusion target \\
\hline
\end{tabular}

equation (2): Exclusion $=(1-e) \cdot[i+(1-i) \cdot h]$. Parameter $h$ represents the base rate in the exclusion condition.

Before the evaluation phase the participants were asked to complete the stems using three-syllable words, no plurals, no proper nouns, present tense for verbs, in $30 \mathrm{~s}$.

\section{Randomization}

Each participant was assigned to sevoflurane group A or propofol group B and also to one of the 3 groups of Table I with the closed envelop method and thus was presented with a different combination of the 3 lists of words. There was also group C of the non-anaesthetised volunteers, who were also randomly assigned to one of the 3 groups of our counterbalancing scheme.

\section{Statistical analysis}

For each participant we calculated the proportion of correct answers over the sum of the presented words (hit rate) and the percentage of correct responses over the sum of distractor words (base rate). Based on these results we estimated the mean explicit memory score (E) and the mean implicit memory score (I) for the three BIS categories $(21-40,41-60,61-80)$ and also for propofol and sevoflurane, separately.

We performed normality and equal variances t-ests for quantitative variables in order to decide when to apply parametric and when to resort to non-parametric $t$-ests. For negative memory values we applied the truncation method and they were set equal to zero. Such values were few and the case of normality was not affected. If a participant heard fewer than 3 words in any condition he was excluded from the particular condition.

We performed one-tailed $t$-tests to check that the calculated memory scores differed from zero. We used paired $t$-tests or the non-parametric equivalent Wilcoxon test to compare mean memory scores and the ANOVA analysis of variance or the corresponding non-parametric Kruskal-Wallis test to compare memory scores to other factors. For post hoc (multiple comparisons) we used Bonferroni tests (i.e. testing gender, education, severity of surgery, etc.)

We used the correlation coefficients of Pearson (for normally distributed variables) and/or Spear- 
man to test possible correlations between memory and quantitative variables (age, duration of anaesthesia/surgery). Correlation tests were also performed (Pearson/Spearman) between memory scores and vital signs or breathing variables $\left(\mathrm{EtCO}_{2}\right.$, $\mathrm{SatO}_{2}$ ). Further tests were performed on participants with extreme (breathing and vital signs) values and those without ( $t$-tests).

The level of statistical significance was set at $p<0.05$ for all tests. The average power of the study (given the available sample size per test and for predefined $a=0.05$ ) was calculated to be 0.88 , which is satisfactory.

\section{Results}

Two participants from the sevoflurane group, 5 from the propofol group and 5 non-anaesthetised were excluded from the study either due to technical problems, accidental or non-accidental deviations from the protocol, or because they were lost to follow-up.

Table II shows subjects' demographic data, operation data, and the retention interval.

Twenty-four participants underwent surgery of moderate (e.g. arthroscopy, wall hernia repair, thy- roidectomy, etc.) and 69 of major surgical grade (e.g. spinal fusion, hysterectomy, laparotomy, etc.).

Table III compares E and Table IV I for the different BIS categories of participants anaesthetised with propofol and sevoflurane. Table $V$ compares $E$ and I of the anaesthetised (of each BIS category) and the non-anaesthetised participants.

No participant manifested explicit recall according to the 5-question interview. The contribution of explicit memory was not significant in any BIS group $(p>0.05)$. Implicit memory was preserved in participants with BIS 41-60 ( $p=0.001)$ and 61-80 $(p=0.008)$ and was absent in those with BIS 21-40 $(p=0.09)$. E was comparable between anaesthetised participants of all three BIS categories $(p=1.00)$, but was not comparable between anaesthetised and non-anaesthetised subjects $(p<0.001)$. I was comparable between participants with BIS (a) 41-60 and 61-80 ( $p=0.067$ ), (b) 41-60 and the nonanaesthetised ( $p=1.00$ ), and (c) 61-80 and the nonanaesthetised ( $p=0.476$ ), but was not comparable between BIS groups (a) 21-40 and 41-60 ( $p<0.001$ ), (b) 21-40 and 61-80 ( $p=0.017)$ and (c) 21-40 and the non-anaesthetised $(p<0.001)$. We found a statistically significant difference between I before inci-

Table II. Participants' demographic data, operation data and retention interval

\begin{tabular}{|lcccc|}
\hline Parameter & $\begin{array}{c}\text { Anaesthetised } \\
\text { with sevoflurane }\end{array}$ & $\begin{array}{c}\text { Anaesthetised } \\
\text { with propofol }\end{array}$ & Non-anaesthetised & Value of $p$ \\
\hline$n$ & 48 & 45 & 45 & 0.142 \\
\hline Age [years] & $43.13 \pm 11.50$ & $38.96 \pm 11.37$ & $40.16 \pm 7.65$ & $19 / 26$ \\
\hline Sex: male/female & $17 / 31$ & $14 / 31$ & $13.33 \pm 1.90$ & 0.122 \\
\hline Educational level [years] & $13.1 \pm 1.75$ & $13.42 \pm 1.93$ & 0.672 \\
\hline Duration of anaesthesia [h] & $138.77 \pm 52.47$ & $141.89 \pm 64.06$ & & 0.802 \\
\hline Duration of surgery [h] & $111.58 \pm 42.06$ & $114.89 \pm 53.83$ & & 0.431 \\
\hline Retention interval [h] & $26.00 \pm 2.85$ & $25.53 \pm 3.27$ & & \\
\hline
\end{tabular}

Data expressed as mean \pm standard deviation (SD)

Table III. Explicit memory score for different BIS categories and anaesthetic agents

\begin{tabular}{|lccccc|}
\hline & E for BIS 21-40 & E for BIS 41-60 & E for BIS 61-80 & E before incision & E after incision \\
\hline Propofol & $0.055 \pm 0.087$ & $0.046 \pm 0.083$ & $0.066 \pm 0.089$ & $0.056 \pm 0.127$ & $0.045 \pm 0.084$ \\
\hline Sevoflurane & $0.025 \pm 0.042$ & $0.035 \pm 0.047$ & $0.004 \pm 0.015$ & $0.064 \pm 0.122$ & $0.030 \pm 0.043$ \\
\hline Value of $p$ & 0.06 & 0.437 & 0.034 & 0.829 & 0.337 \\
\hline
\end{tabular}

Data expressed as mean \pm standard deviation (SD), E- explicit memory score

Table IV. Implicit memory score, for different BIS categories and anaesthetic agents

\begin{tabular}{|lccccc|}
\hline & I for BIS 21-40 & I for BIS 41-60 & I for BIS 61-80 & I $_{\text {B.I. }}$ & I A.I. \\
\hline Propofol & $0.016 \pm 0.053$ & $0.082 \pm 0.079$ & $0.112 \pm 0.140$ & $0.046 \pm 0.101$ & $0.070 \pm 0.064$ \\
\hline Sevoflurane & $0.009 \pm 0.025$ & $0.125 \pm 0.095$ & $0.046 \pm 0.088$ & $0.038 \pm 0.094$ & $0.113 \pm 0.071$ \\
\hline Value of $p$ & 0.439 & 0.23 & 0.15 & 0.782 & 0.005 \\
\hline
\end{tabular}

Data expressed as mean \pm standard deviation (SD); I - implicit memory score, $I_{\text {B.I. }}$ - implicit memory score before incision, I A.I. $_{-}$implicit memory score after incision 
Table V. Explicit memory score and implicit memory score for all BIS categories of anaesthetised (regardless of anaesthetic agent) and non-anaesthetised participants

\begin{tabular}{|c|c|c|c|c|}
\hline \multirow{2}{*}{$\begin{array}{l}\text { Parameter } \\
E\end{array}$} & \multicolumn{2}{|c|}{ Anaesthetised } & \multirow{2}{*}{$\begin{array}{c}\text { Non-anaesthetised } \\
0.114 \pm 0.107\end{array}$} & \multirow{2}{*}{$\begin{array}{c}\text { Value of } p \\
<0.001\end{array}$} \\
\hline & BIS 21-40 & $0.039 \pm 0.068$ & & \\
\hline & BIS 41-60 & $0.040 \pm 0.067$ & & $<0.001$ \\
\hline & BIS 61-80 & $0.036 \pm 0.071$ & & $<0.001$ \\
\hline \multirow[t]{3}{*}{ 1 } & BIS 21-40 & $0.012 \pm 0.040$ & $0.089 \pm 0.077$ & $<0.001$ \\
\hline & BIS 41-60 & $0.096 \pm 0.078$ & & 1.000 \\
\hline & BIS 61-80 & $0.045 \pm 0.087$ & & 0.476 \\
\hline
\end{tabular}

Data expressed as mean \pm standard deviation (SD); E- explicit memory score, I - implicit memory score

sion $\left(\mathrm{I}_{\mathrm{B} . \mathrm{I}}\right)$ and I after incision $\left(\mathrm{I}_{\mathrm{A} . \mathrm{I}}\right)(p=0.004)$. There was no statistically significant difference between the base rates in the inclusion and the exclusion condition $(p=0.127)$.

The type of anaesthetic drug seemed to have an effect on memory. E was insignificant in all BIS categories for both anaesthetic drugs. There was a statistically significant difference between I and $I_{\text {A.I. }}$ in the propofol and the sevoflurane group in BIS category $41-60$ ( $p=0.023$ and 0.005 respectively), with propofol depressing implicit memory more than sevoflurane in both cases.

Age had a very weak positive correlation with I for BIS 41-60 ( $p=0.019)$. The duration of surgery had a weak positive correlation with $E$ for BIS $41-60(p=0.049)$. Educational level did not seem to correlate with memory $(p>0.1)$, while sex seemed to correlate with $\mathrm{E}$ for $\mathrm{BIS}>60\left(\mathrm{E}_{\text {male }}>\mathrm{E}_{\text {female, }}\right.$, $p=0.076)$ and $I_{\text {B.I. }}\left(I_{\text {B.I. male }}>I_{\text {B.I female, }} p=0.016\right)$.

Major surgical grade seemed to enhance participants' I for BIS 41-60 $(p=0.04)$ and $\mathrm{I}_{\mathrm{A} . \mathrm{I}}$. $(p=0.02)$, and suppress E for BIS 41-60 ( $p=0.027)$.

Correlation tests were performed between the vital signs (even extreme haemodynamic values) and breathing variables recorded during the operation and each type of memory but they did not provide clear and strong conclusions.

\section{Discussion}

According to our findings general anaesthesia regardless of agent significantly suppresses explicit memory. Implicit memory is preserved during light and adequate anaesthesia to a degree comparable to the absence of anaesthesia. Both types of memory disappear during deep anaesthesia. Implicit memory of anaesthetised participants is better preserved with sevoflurane than with propofol for BIS 41-60. Surgical incision and also surgery of major degree seemed to enhance implicit memory performance for both agents despite recent evidence that implicit learning is not sensitive to stress [28].

The base rates in our study are much lower than in other studies [10-13, 29]. This could possibly be explained by the fact that we used 3-syllable words and as a peculiarity of the Greek language that offers many alternatives for word stem completion.

None of our participants reported conscious recall of any intraoperative events. The overall (regardless of BIS) E in our study was comparable to the results of older studies $[10,28]$. We found no explicit recall in any group of anaesthetised participants in accordance with the literature [30]. We did not find any contribution of explicit memory even in the group of lightly anaesthetised participants either. Some previous studies have suggested the existence of a weak form of explicit memory that can occur even in the absence of conscious recall in light planes of anaesthesia [12, 13]. We, like Stapleton and Andrade and Chaves [13, 28], believe that this is a phenomenon of subjects' manipulation by the investigator's instructions on how to respond. Subjects tend to use or avoid familiar words or special terms according to the instructions given in the inclusion and exclusion conditions.

The choice of anaesthetic drug (propofol or sevoflurane) did not seem to have a significant impact on explicit memory according to our findings. Our E for the participants lightly anaesthetised with propofol is similar to that of Stapleton's and Andrade's study [13] as well as Lubke's et al.'s study on trauma patients [10]. Our finding that in the propofol group there is no evidence of explicit memory for the adequately and deeply anaesthetised participants is again in concert with the results of older studies $[17,18]$.

We found no contribution of explicit memory in the sevoflurane group of lightly anaesthetised participants either. Other authors such as Chaves [29] and Lubke et al. [11] studying isoflurane demonstrated the occurrence of explicit memory in the group of lightly anaesthetised patients, but this could be due to moments of lighter anaesthesia. Our $E$ in the sevoflurane group of adequately and deeply anaesthetised patients is similar to that of Chaves' study using isoflurane [29].

To compare our finding that implicit memory is preserved during adequate anaesthesia to those of 
older studies we focused on studies of anaesthetised participants having some type of hypnotic state monitoring and being assessed with the PDP.

There are several studies about implicit memory during general anaesthesia with propofol. Münte reported intraoperative preservation of implicit memory as indicated by an increased postoperative reading speed for stories presented during light to moderate but not deep hypnotic stages as evidenced by Narcotrend [31]. Deeprose reported significant implicit memory during adequate anaesthesia with propofol in two studies $[15,16]$. Kerssens, on the other hand, using category exemplar generation and the PDP $[15,16]$ and Struys et al. [32] using intraoperative suggestions failed to show the preservation of implicit memory at adequate anaesthesia level (BIS 41-60) with propofol, but both studies had differences in methodology compared to ours. Hadzidiakos et al. in their study [14] despite using Buchner's model also found no memories for auditory information presented during propofolremifentanil anaesthesia, but he routinely used midazolam, and the median BIS was 32.5.

There are a few studies using sevoflurane. Renna et al. indicated the persistence of implicit memory in patients receiving $1.2 \%$ but not $1.5 \%$ and $2 \%$ end tidal sevoflurane regardless of BIS [20]. Kerssens et al. [21] and Stonell et al. using Buchner's model [22] reported implicit memory during adequate levels of general anaesthesia with sevoflurane. Aceto, studying sevoflurane and isoflurane with the use of midlatency auditory evoked potentials (MEPs), found preservation of automatic memory for latencies indicating light anaesthesia under different anaesthetic regimens but MEPs do not correspond directly to the BIS value [33]. Kristoffer, on the other hand, found that sevoflurane anaesthesia prevents auditory perceptual learning, but he used an auditory perceptual learning paradigm (tone discrimination) relying on different brain influences than a word stem completion task.

It is obvious that different studies have different and sometimes opposite results about memory during general anaesthesia with propofol and sevoflurane. The physiological role of persistent implicit memory in anaesthesia, and the possible practical and psychological implications for people under general anaesthesia, still remain a mystery. More studies are required in order to establish guidelines about the harmless administration of anaesthesia with regard to memory.

In conclusion, implicit memory persists during general anaesthesia for elective surgery even in adequate (but not deep) hypnotic states. Although sevoflurane seems to depress implicit memory to a greater yet statistically comparable degree to propofol, it seems to affect the implicit memory of adequately anaesthetised subjects less than propofol.

\section{Acknowledgments}

This work was not supported financially, is not under any research contract and the authors have no conflicts of interest.

\section{References}

1. Veselis RA, Pryor KO. Propofol amnesia: what's going on in the brain? In: Suppressing the mind: anesthetic modulation of memory and consciousness. Hudits A, Pearce R (eds.). $1^{\text {st }}$ ed. Human Press, New York 2010; 215-43.

2. Ghoneim MM. Drugs and human memory (part 2). Clinical, theoretical and methodologic issues. Anesthesiology 2004; 100: 987-1002.

3. Liu W, Thorp T, Graham S, Aitkenhead. A. Incidence of awareness with recall during general anaesthesia. Anaesthesia 1991; 46: 435-7.

4. Sandin R, Enlund G, Samuelsson P, Lennmarken C. Awareness during anaesthesia: a prospective case study. Lancet 2000; 355: 707-11

5. Sebel P, Bowdle T, Ghoneim M, et al. The incidence of awareness during anesthesia: a multicenter United States Study. Anesth Analg 2004; 99: 833-9.

6. Ghoneim MM. On memory, general anesthesia, and sleep. Anesthesiology 2010; 113: 1480-1.

7. Merikle PM, Daneman M. Memory for unconsciously perceived events: evidence from anesthetized patients. Conscious Cogn 1996; 5: 525-41.

8. Andrade J. Learning during anaesthesia: a review. Br J Psychol 1995; 86: 479-506.

9. Munte S, Schmidt M, Meyer M, et al. Implicit memory for words played during isoflurane- or propofol-based anesthesia: the lexical decision task. Anesthesiology 2002; 96: 588-94.

10. Lubke G, Kerssens C, Phaf H, Sebel P. Dependence of explicit and implicit memory on hypnotic state in trauma patients. Anesthesiology 1999; 90: 670-80.

11. Lubke G, Kerssens C, Gershon R, Sebel P. Memory formation during general anesthesia for emergency caesarean sections. Anesthesiology 2000; 92: 1029-34.

12. Kerssens C, Lubke G, Klein J, Van der Woerd A, Bonke B. Memory function during propofol and alfentanil anesthesia: predictive value of individual differences. Anesthesiology 2002; 97: 382-9.

13. Stapleton C, Andrade J. An investigation of learning during propofol sedation and anesthesia using the process dissociation procedure. Anesthesiology 2000; 93: 1418-25.

14. Hadzidiakos D, Horn D, Buchner A, Rehberg B. Analysis of memory formation during general anesthesia (propofol/remifentanyl) for elective surgery using the processdissiciation-procedure. Anesthesiology 2009; 111: 1010-5.

15. Deeprose C, Andrade J, Varma S, Edwards N. Unconscious learning during surgery with propofol anaesthesia. $\mathrm{Br}$ J Anaesth 2004; 92: 171-7.

16. Deeprose C, Andrade J, Harrison N, Edwards N. Unconscious auditory priming during surgery with propofol and nitrous oxide anaesthesia: a replication. Br J Anaesth 2005; 94: $57-62$

17. Kerssens C, Klein J, Van Der Woerd A, Bonke B. Auditory information processing during adequate propofol anesthesia monitored by electroencephalogram bispectral index. Anesth Analg 2001; 92: 1210-4.

18. Kerssens C, Takashi O, Sebel P. No evidence of memory function during anesthesia with propofol or isoflurane with close control of hypnotic state. Anesthesiology 2005; 102: 57-62. 
19. Dobrunz UE, Jaeger K, Vetter G. Memory priming during light anaesthesia with desflurane and remifentanil anaesthesia. Br J Anaesth 2007; 98: 491-6.

20. Renna M, Lang E, Lockwood G. The effect of sevoflurane on implicit memory: a double-blind, randomised study. Anaesthesia 2000; 55: 634-40.

21. Kerssens C, Galther JR, Sebel P. Preserved memory function during bispectral index-guided anesthesia with sevoflurane for major orthopaedic surgery. Anesthesiology 2009; 111: 518-24.

22. Stonell CA, Leslie K, He C, Lee L. No sex differences in memory formation during general anesthesia. Anesthesiology 2006; 105: 920-6.

23. Kristoffer CA, Albrecht E, Tartaglia EM, Farron A, Soom P, Herzog MH. Anesthesia prevents auditory perceptual learning. Anesthesiology 2009; 111: 1010-5.

24. Flouda L, Lotis C, Matsota P, et al. Method for the evaluation of the function of implicit memory of Greek patients under general anaesthesia. Acta Anaesthesiologica Hellenica 2008; 41: 141-51.

25. Jacoby L. A process dissociation framework: separating automatic from intentional uses of memory. J Mem Lang 1991; 30: 513-41.

26. Buchner A, Erdfelder E, Vaterrodt-Plunnecke B. Toward unbiased measurement of conscious and unconscious memory processes within the process dissociation framework. J Exp Psychol Gen 1995; 124: 137-60.

27. Brice DD, Hetherington RR, Utting JE. A simple study of awareness and dreaming during anaesthesia. Br J Anaesth 1970; 42: 535-42.

28. Luethi M, Meier B, Sandi C. Stress effects on working memory, explicit memory, and implicit memory for neutral and emotional stimuli in healthy men. Front Behav Neurosci 2009; 2: 1-9.

29. Iselin-Chaves IA, Willems SJ, Jermann FC, Forster A, Adam SR, Van der Linden M. Investigation of implicit memory during Isoflurane anesthesia for elective surgery using the process dissociation procedure. Anesthesiology 2005; 103: 925-33.

30. Glass P, Bloom M, Kearse L, Rosow C, Sebel P, Manberg P. Bispectral analysis measures sedation and mem ory effects of propofol, midazolam, isoflurane, and alfentanil in healthy volunteers. Anesthesiology 1997; 86: 836-47.

31. Münte S, Münte T, Grotkamp J, et al. Implicit memory varies as a function of hypnotic electroencephalogram stage in surgical patients. Anesth Analg 2003; 97: 132-8.

32. Struys M, Versichelen L, Byttebier G, Mortier E, Moerman A, Rolly G. Clinical usefulness of the bispectral index for titrating propofol target effect-site concentration. Anaesthesia 1998; 53: 4-12.

33. Aceto P, Valente A, Gorgoglione E, Adducci E, De Cosmo G. Relationship between awareness and middle latency auditory evoked responses during surgical anaesthesia. $\mathrm{Br}$ J Anaesth 2003; 90: 630-5. 